\title{
Epithelial apoptosis in the initiation of lung fibrosis
}

\author{
B.D. Uhal
}

\begin{abstract}
Apoptosis in the lung is a relatively new area of research that is receiving increasing attention. A growing body of evidence indicates that apoptosis is controlled in a tissue- and cell type-specific manner; for this reason, the regulation of apoptosis in specific cell types of the lung is currently an area of intensive research [1]. This article provides evidence supporting the viewpoint that apoptosis of alveolar epithelial cells is a key event in the initiation of fibrotic lesions, and will discuss a working hypothesis to explain possible mechanisms by which epithelial apoptosis can lead to lung fibrogenesis.
\end{abstract}

\section{Why study apoptosis?}

Recent evidence implicates important roles for apoptosis in animal models of lung injury/repair or remodelling and in lung biopsies from patients with a variety of lung disorders. For example, apoptosis plays a critical role in postnatal lung development [2] and in the normal resolution of lung inflammation by the regulated removal of unneeded cells such as granulocytes, without the release of damaging histotoxins [3]. Dexamethasone has been known for many years to induce apoptosis in some leukocyte subsets, and apoptosis is an important mechanism underlying the anti-inflammatory action of this and other glucocorticoids [4]. Considerable literature now supports a role for apoptosis in the remodelling of lung tissue after acute lung injury, both for the clearance of excess epithelial stem cells after hyperplastic repair [5] and for the normal removal of excess mesenchymal cells from resolving lesions [6]. Extensive apoptosis is found in the lungs of patients with idiopathic pulmonary fibrosis (IPF) [7, 8] and in animals of this disease [9, 10]. Recent evidence also suggests a role for apoptosis in the tissue remodelling associated with chronic pulmonary hypertension [11] and with chronic obstructive pulmonary disease [12].

\section{Apoptosis in the alveolar epithelium}

The author's laboratory has focused on the alveolar epithelial cell (AEC) because of its many important functions, which include, but are not limited to, the synthesis and secretion of pulmonary surfactant, local antioxidant defence, local immunomodulation, synthesis and turnover of extracellular matrix molecules, and high proliferative capacity for the repair of damaged epithelium [13]. To study these cells and their role(s) in lung injury and fibrosis, Wistar rat models and primary cell cultures of AECs isolated from the same animals are used.

Correspondence: B.D. Uhal, Dept of Physiology, Michigan State University, 3185 Biomedical \& Physical Sciences Building, East Lansing, MI48824-330, USA. Fax: +1 5173555125. E-mail: uhal@, msu.edu

This work was supported by PHS HL-45136, by the American Heart Association and by the MSU Foundation.
The rapidly growing list of physiologically relevant molecules that are now known to induce apoptosis of AECs includes oxidants generated during the oxidative burst [14], activators of the "death receptor" Fas (apoprotein 1/CD95) [15], tumour necrosis factor (TNF)- $\alpha$ [16], the antineoplastic agent bleomycin [9], the popular but pneumotoxic antiarrhythmic agent amiodarone [17] (fig. 1), noradrenaline (NA) [18] and angiotensin (ANG)II [19, 20]. The latter is particularly important because in vitro studies have demonstrated that ANGII, synthesised de novo by the AEC, is required as a mediator of the apoptotic response to Fas activation, to TNF- $\alpha$, amiodarone, NA [15-18], and possibly other inducers [9]. Importantly, agents capable of blocking the ANGII production or receptor interaction on AECs can thus effectively block the apoptosis.

\section{Apoptosis in lung fibrosis}

With specific regard to pulmonary fibrosis, complimentary lines of evidence suggest that apoptosis of specific cell types is involved in both the pathogenesis and the resolution of fibrotic lesions. A prominent feature of the fibrotic lung is proliferation and accumulation of mesenchymal cells. These include abnormal fibroblast populations that emerge in the interstitium but may migrate into the alveolar spaces [21], and endothelial cells that evolve during neovascularisation of nascent fibrotic foci [6]. In patients who recover from fibrotic lung disease, these excess cells are removed by processes, which, although not completely understood, likely include apoptosis $[5,6]$.

A number of studies now support the notion that apoptosis contributes to the pathogenesis of lung fibrosis as well, particularly in the initiation of fibrotic foci. In mouse lung, intratracheal instillation of an antibody that activates the receptor Fas, induced apoptosis of bronchial cells and AECs (both of which express Fas constitutively) and initiated a fibrotic response that was detectable 1 week later [22]. The same research group showed that the induction of lung fibrosis by intratracheal instillation of bleomycin is associated with upregulation of Fas on the epithelium and concomitant induction of epithelial apoptosis as a prelude to fibrogenesis [10]. These results support the hypothesis proposed years earlier by WITSCHI [23] and ADAMSON et al. [24], which suggests that the destruction of the healthy alveolar epithelium, in itself, creates a profibrotic microenvironment that initiates a nascent fibrotic focus. Consistent with that interpretation, the author's study of lung biopsies from patients with IPF has shown that nascent fibrotic foci colocalise with apoptotic or absent alveolar epithelium [7].

Recent data suggest that specific blockade of apoptosis, in itself, is sufficient to prevent the fibrotic response that would otherwise follow. In a study of lung fibrogenesis in rats [9], the author's group found that the accumulation of lung collagens after intratracheal administration of bleomycin could be blocked with equal potency by the angiotensin-converting 
enzyme (ACE) inhibitor captopril or by intraperitoneal injections of ZVADfmk, a broad spectrum inhibitor of caspases (cysteine proteases) required for the induction of apoptosis. These results were confirmed the following year by KUWANO et al. [25] through a similar study of bleomycininduced lung fibrosis in mice, in which the caspase inhibitor was administered by aerosol. Regardless of the route of drug delivery, the blockade of collagen deposition in vivo by a specific inhibitor of apoptosis suggests that the fibrotic response is secondary to the apoptotic death of certain lung cell types, and is consistent with the theories put forth by WiTsCHI [23] and ADAMSON et al. [24]

\section{Angiotensin and lung fibrosis}

The blockade of collagen deposition by the ACE inhibitor captopril also confirmed earlier studies by MOLTENI et al. [26] and WARD et al. [27], who had shown years before that ACE inhibitors and related compounds had potent antifibrotic potential in several animal models. In light of the in vitro findings that ACE inhibitors and ANG receptor antagonists also potently block apoptosis of AECs [15-17, 28], it was hypothesised that at least part of the antifibrotic potential of these agents can be attributed to their ability to prevent the apoptotic death of the epithelial layer. In support of this premise, antagonists of the ANG receptor AT1 can block apoptosis of AECs in response to purified ANGII [29] or bleomycin in vitro [30], and amiodarone both in vitro (fig. 1) and in vivo [31]; furthermore, the AT1 antagonist losartan blocked the development of amiodarone-induced lung fibrosis [31].

These data suggest a critical role for ANG generation and interaction with receptor AT1 in the induction of AEC apoptosis and subsequent lung fibrogenesis. With regard to human lung, the author has also shown that myofibroblasts derived from lung biopsies obtained from patients with IPF produce and secrete ANG peptides, and on this basis it was hypothesised that local ANG production is key in both initiating and "propogating" the fibrotic focus [20].

\section{Working hypothesis}

The author's current work is addressing the mechanisms of

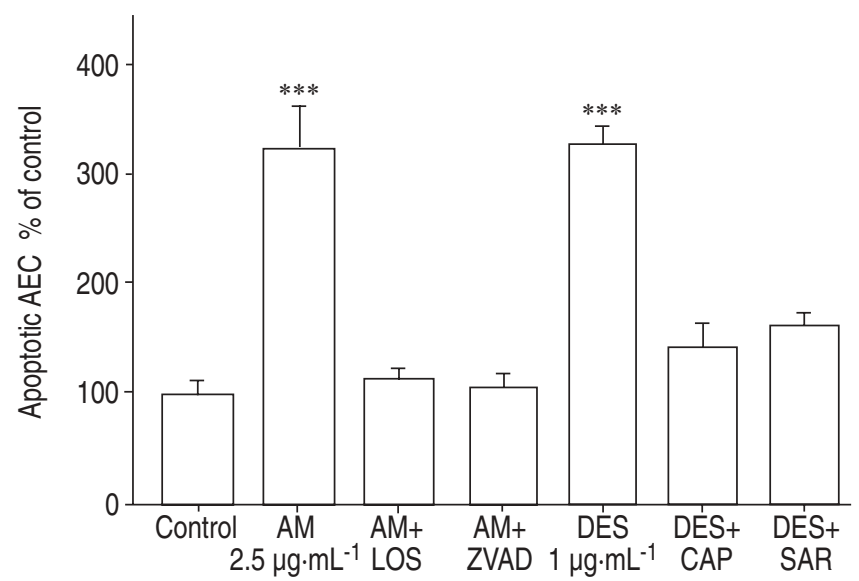

Fig. 1.-Amiodarone (AM) or its metabolite desethylamiodarone (DES) induce apoptosis in primary cultures of alveolar epithelial cells (AECs) that can be abrogated by captopril (CAP), the angiotensin receptor antagonists losartan (LOS) or saralasin (SAR) or the caspase inhibitor ZVADfmk (ZVAD). ***: $\mathrm{p}<0.001$ versus control.

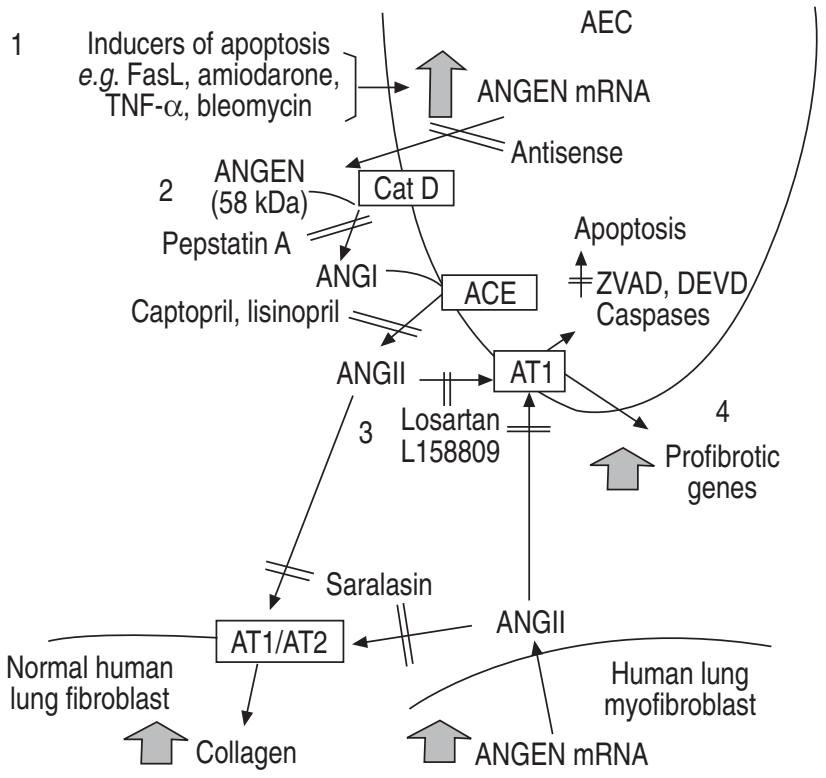

Fig. 2.-1) Fibrogenic agents induce apoptosis and upregulate angiotensinogen (ANGEN) synthesis by alveolar epithelial cells. Blockers of angiotensin (ANG)II synthesis (antisense or angiotensin-converting enzyme inhibitors) block alveolar epithelial cell (AEC) apoptosis. 2) Aspartyl proteases such as Cathepsin D (Cat D) are activated by apoptosis inducers (fibrogenic agents); blockage by pepstatin A prevents AEC apoptosis. 3) ANG receptor AT1 mediates AEC apoptosis in response to ANGII produced by autocrine mechanisms or by myofibroblasts. AT1 blockers prevent AEC apoptosis and fibrosis. 4) Activation of AT1 induces profibrotic gene expression by AECs during apoptosis. Blockage of AT1 prevents apoptosis and profibrotic gene expression. FasL: Fas ligand; TNF: tumour necrosis factor; mRNA: messenger ribonucleic acid; ZVAD: $N$-benzylcarboxy-Val-Ala-Aspfluoromethylketone; DEVD: Asp-Glu-Val-Asp-fluoromethylketone. $=$ : known points of blockade.

apoptosis-related ANG production by the AEC and adjacent myofibroblasts, and its pathophysiological consequences in the context of fibrogenesis. To summarise this working hypothesis see figure 2 .

These data provide a molecular mechanism that directly links cell death in the alveolar epithelium to local production of angiotensin, a molecule with well-documented and multiple profibrotic actions. They also provide an explanation for the documented ability of alveolar epithelial cell inhibitors or angiotensin-receptor antagonists to prevent alveolar epithelial cell apoptosis and experimental pulmonary fibrosis in several animal models. One attractive feature of this model is its ability to explain fibrogenesis of unknown origin, regardless of whether the initial stimuli were chemicals, inflammatory mediators or other endogenous inducers of apoptosis.

\section{References}

1. Fine A, Janssen-Heininger Y, Soultanakis RP, Swisher S, Uhal BD. Apoptosis in lung pathophysiology. Am J Physiol 2000; 279: L423-L427.

2. Schittney J, Djonov V, Fine A, Burri PH. Programmed cell death contributes to postnatal lung development. Am J Respir Cell Mol Biol 1998; 18: 786-793.

3. Haslett C. Granulocyte apoptosis and its role in the resolution and control of lung inflammation. Am J Respir Crit Care Med 1999; 160: S5-S11.

4. Meagher L, Cousin JM, Seckl R, Haslett C. Opposing effects of glucocorticoids on the rate of apoptosis in neutrophilic and eosinophilic granulocytes. J Immunol 1996; 156: 4422 4428 . 
5. Bardales RH, Xie SS, Schaefer RF, Hsu S. Apoptosis is a major pathway responsible for the resolution of type II pneumocytes in acute lung injury. Am J Pathol 1997; 149: 845-852.

6. Polunovsky V, Chen B, Henke C, et al. Role of mesenchymal cell death in lung remodeling after injury. J Clin Invest 1993; 92: 388-397.

7. Uhal BD, Joshi I, Ramos C, Pardo A, Selman M. Alveolar epithelial cell death adjacent to underlying myofibroblasts in advanced fibrotic human lung. Am J Physiol 1998; 275: L1192-L1199.

8. Kuwano K, Kunitake R, Kawasaki M, et al. p21 and p53 expression in association with DNA strand breaks in idiopathic pulmonary fibrosis. Am J Respir Crit Care Med 1996; 154: 477-483.

9. Wang R, Ibarra-Sunga O, Pick R, Uhal BD. Abrogation of bleomycin-induced epithelial apoptosis and lung fibrosis by captopril or by a caspase inhibitor. Am J Physiol 2000; 279: L143-L151.

10. Hagimoto N, Kuwano K, Nomoto Y, Kunitake R, Hara N. Apoptosis and expression of FAS/FAS ligand mRNA in bleomycin-induced pulmonary fibrosis in mice. Am J Respir Cell Mol Biol 1997; 16: 91-101.

11. Durmowicz AG, Stenmark KR. Mechanisms of structural remodeling in chronic pulmonary hypertension. Pediatr Rev 1999; 20: e91-e102.

12. Segura L, Pardo A, Gaxiola M, Uhal BD, Becerril C, Selman $\mathrm{M}$. Upregulation of gelatinases A and B, collagenases 1 and 2 , and increased parenchymal cell death in COPD. Chest 2000; 117: 684-694.

13. Uhal BD. Cell cycle kinetics in the alveolar epithelium. Am J Physiol 1997; 272: L1031-L1045.

14. Kazzaz JA, Xu J, Palai TA, Mantell L, Fein AM, Horowitz S. Cellular oxygen toxicity. Oxidant injury without apoptosis. J Biol Chem 1996; 271: 15182-15186.

15. Wang R, Zagariya A, Ang E, Ibarra-Sunga O, Uhal BD. Fas-induced apoptosis of alveolar epithelial cells requires angiotensin II generation and receptor interaction. Am J Physiol 1999; 277: L1245-L1250.

16. Wang R, Alam G, Zagariya A, et al. Apoptosis of lung epithelial cells in response to TNF- $\alpha$ requires angiotensin II generation de novo. J Cell Physiol 2000; 185: 253-259.

17. Bargout R, Jankov A, Dincer E, et al. Amiodarone induces apoptosis in human and rat alveolar epithelial cells in vitro. Am J Physiol 2000; 278: L1039-L1044.

18. Dincer H, Gangopadhyay N, Wang R, Uhal BD. Norepinephrine induces alveolar epithelial apoptosis mediated by $\alpha$, $\beta$ and angiotensin receptor activation. Am J Physiol Lung Cell Mol Physiol 2001; 281: L624-L630.

19. Wang R, Zagariya A, Ibarra-Sunga O, et al. Angiotensin II induces apoptosis in human and rat alveolar epithelial cells. Am J Physiol 1999; 276: L885-L889.

20. Wang $\mathrm{R}$, Ramos $\mathrm{C}$, Joshi I, et al. Human lung myofibroblast-derived inducers of alveolar epithelial apoptosis identified as angiotensin peptides. Am J Physiol 1999; 277: L1158-L1164.

21. Selman M. Pulmonary fibrosis: human and experimental disease. In: Rojkind M, ed. Connective Tissue in Health and Disease. Boca Raton, CRC Press Inc., 1990; pp. 123188.

22. Hagimoto N, Kuwano K, Miyazaki H, et al. Induction of apoptosis and pulmonary fibrosis in mice in response to ligation of FAS antigen. Am J Respir Cell Mol Biol 1997; 17: 272-278.

23. Witschi H. Responses of the lung to toxic injury. Environ Health Perspect 1990; 85: 5-13.

24. Adamson IYR, Young L, Bowden DH. Relationship of alveolar epithelial injury and repair to the induction of pulmonary fibrosis. Am J Pathol 1988; 130: 377-383.

25. Kuwano K, Kunitake R, Maeyama T, et al. Attenuation of bleomycin-induced pneumopathy in mice by a caspase inhibitor. Am J Physiol 2001; 280: L316-L325.

26. Molteni A, Ward W, Ts'ao C, Solliday N, Dunne M. Monocrotaline-induced pulmonary fibrosis in rats: amelioration by captopril and penicillamine. Proc Soc Exp Biol Med 1985; 180: 112-120.

27. Ward W, Molteni A, Ts'ao C, Hinz J. The effect of captopril on benign and malignant reactions in irradiated rat skin. Br J Radiol 1990; 63: 349-354.

28. Uhal BD, Gidea C, Bargout R, et al. Captopril inhibits apoptosis in human lung epithelial cells: a potential antifibrotic mechanism. Am J Physiol 1998; 275: L10131017.

29. Papp M, Li X, Zhuang J, Wang R, Uhal BD. Angiotensin receptor subtype $\mathrm{AT}_{1}$ mediates alveolar epithelial cell apoptosis in response to ANGII. Am J Physiol 2002; 282: L713-L718.

30. Li X, Zhuang H, Soledad-Conrad V, Zhang J, Uhal BD. Bleomycin-induced apoptosis of alveolar epithelial cells requires angiotensin synthesis de novo. Am J Physiol 2003; 284: L501-L507.

31. Uhal BD, Wang R, Laukka J, Zhaung J, Soledad-Conrad V, Filippatos G. Inhibition of amiodarone-induced lung fibrosis but not alveolitis by angiotensin system antagonists. Pharmacol Toxicol 2003; 92: 81-87. 